\title{
A Stepwise Approach to the Management of Heart Failure and its Comorbidities
}

Felix J. Rogers, DO; Zeinab Saghir, DO

From Henry Ford Wyandotte Downriver Cardiology in Brownstown, Michigan (Dr Rogers) and Beaumont Health in Trenton,

Michigan (Dr Saghir).

Dr Saghir is a third-year Internal Medicine resident.

Financial Disclosures:

None reported.

Support: None reported.

Address correspondence to

Felix J. Rogers, DO,

Downriver Cardiology Consultants at Henry Ford Health Center, 23050 West Rd, Ste 120, Brownstown, MI 48183-1470.

Email: frogers1@hfhs.org Submitted February 8, 2019; revision received April 1, 2019; accepted April 30, 2019.
Modern medical and technological advances provide highly effective management for the treatment of patients with heart failure with reduced ejection fraction (HFrEF). In this review, the authors propose a 2-step approach to treatment that is straightforward, practical, and thorough. For the patient whose life now includes HFrEF, the physician's first step is to ensure that the patient is taking the 3 key medications ([1] renin-angiotensin inhibitors (angiotensin receptor/neprilysin inhibitors, angiotensin-converting enzyme inhibitors, or angiotensin receptor blockers), [2] $\beta$-blockers, and [3] mineralocorticoid receptor antagonists) recommended in guideline-directed doses to attain comprehensive receptor blockade. Significant coexisting medical issues are also characteristic in patients with HFrEF. Therefore, the physician's second step is to address the comorbidities of heart failure to fulfill comprehensive patient care. This review presents evidence to implement the management of $\mathrm{HFrEF}$ and heart failure comorbidities that will reduce cardiac mortality and hospitalization and to avoid treatments that are of no benefit or may cause harm.

J Am Osteopath Assoc. 2020;120(2):90-99

doi:10.7556/jaoa.2020.007

Keywords: coronary artery disease, heart failure, heart failure with reduced ejection fraction

he American College of Cardiology (ACC) and the American Heart Association (AHA) guidelines for heart failure (HF) management are now a central aspect of care for patients with HF with reduced ejection fraction (HFrEF). ${ }^{1}$ The HFrEF guidelines are updated at intervals of 5 to 7 years..$^{2-4}$ This review examines evidence from research trials published after the most recent updates to the guidelines ${ }^{2-4}$ and addresses the management of comorbid conditions of high clinical relevance, such as coronary artery disease (CAD), arrhythmias, type 2 diabetes (T2D), and lifestyle issues, including exercise, smoking, and diet. This review emphasizes management of the ambulatory patient from the perspective of the practicing physician and provides evidencebased recommendations to improve patient care in terms of the objective end points of patient survival and rehospitalization rates.

A patient with newly diagnosed HFrEF must now cope with living with this condition for the rest of his or her life. These patients are not alone-an estimated 5.7 million people in the United States have $\mathrm{HF},{ }^{5}$ and roughly half have HFrEF. Heart failure with reduced ejection fraction is a dangerous journey because about half of patients with newly diagnosed HFrEF will die within the first 5 years. ${ }^{5}$ For this reason, it is an apt analogy to consider the physician as an airline pilot at the start of a trip. Like pilots making a preflight checklist to ensure a safe journey for their passengers, physicians can 
make similar use of checklists ${ }^{6}$ and expert guidelines ${ }^{1}$ to yield improvements in performance and outcomes for the patients entrusted to their care.

There are 3 categories of medication for patients with $\mathrm{HF}$ - (1) renin-angiotensin inhibitors (angiotensin receptor/neprilysin inhibitors [ARNIs], angiotensinconverting enzyme inhibitors [ACEIs], or angiotensin receptor blockers [ARBs]), (2) $\beta$-blockers, and (3) mineralocorticoid receptor antagonists (MRAs) — that are proven to increase survival and reduce hospitalization in patients with HFrEF. Taken together, they provide comprehensive receptor blockade, which reduces the production of intrinsic biologic compounds that worsen HF and enhances the activity of naturally occurring proteins that improve cardiovascular function.

For optimal efficacy, each patient needs to be prescribed the correct medication at the proper dose. Nationwide, physicians fall short of this goal; comprehensive surveys of 150 physician practices showed that only $1 \%$ of patients received guideline-directed medical therapy (GDMT) doses of all classes of medication. ${ }^{7}$ Among eligible patients, $27 \%$ were not prescribed renin-angiotensin inhibitors, 33\% were not prescribed $\beta$-blockers, and $67 \%$ were not prescribed MRAs. ${ }^{7}$ In a study $^{8}$ of 11,064 patients with HFrEF, worsening of HF occurred within 1.5 years of incident HF in $17 \%$ of patients. At the time of worsening of $\mathrm{HF}$, only $14 \%$ of patients were using triple therapy, and the majority of these patients were not prescribed GDMT doses. At times of clinical decompensation, adherence to GDMT deteriorated further.

The traditional frontline use of ACEIs has been supplanted. Sacubitril/valsartan, an ARNI, combines the ARB valsartan with the neprilysin inhibitor sacubitril. This ARNI has been shown to be superior to ACEI in the management of class II and III HF. ${ }^{9}$ The 2016 update to the guidelines recommend that patients who are doing well taking an ACEI or ARB should switch to an ARNI if possible. ${ }^{2}$ A 2018 study of sacubitril/valsartan found that this agent was underused, in part because of physician inertia but also because of cost. ${ }^{10}$
Every patient with HF should also be prescribed a $\beta$-blocker, of which only 3 are recommended: carvedilol, metoprolol succinate, and bisoprolol. Patients should also be prescribed an MRA, of which spironolactone is more commonly used than eplerenone. Each of these medications should be used in doses that match GDMT (Table 1).

The second step in systematic management is to address the comorbid conditions of the patient with HFrEF. Physicians should prescribe medications of proven value (class I and II recommendations supported by strong levels of evidence [A or B]) and avoid medications of little or no value or of harm (class IIB and class III, respectively; Table 2).

\section{Methods}

The starting point for this literature review was the 2013 guideline $^{1}$ for the management of patients with HF. The second step was the review of updates to those guidelines since publication. ${ }^{2-4}$ Anemia, hypertension, and sleep disordered breathing were addressed in 2017 as significant comorbidities (Figure 1). We selected the additional comorbid conditions of T2D, arrhythmias, $\mathrm{CAD}$, and lifestyle. Because this review is aimed at physicians treating an ambulatory patient population, citations concerning in-hospital care or patients with ACC stage D HF who were candidates for left ventricular assist devices or heart transplantation were not considered.

The scope of this review is configured in relationship to the guidelines of the ACC/AHA, which are considered to be authoritative. In this context, the recommendations we provide use the comparator terms of "reasonable" or "may be considered" rather than the declarative statement of "should," which is found in the guidelines after authoritative review. The purpose of this review is to assist physicians in clinical decision making. As with the guidelines, our recommendations carry the warning that specific medication doses and clinical applications for recommendations need to be determined based on specific features of the individual patient. 
Table 1.

Examples of Guideline-Based Targets for Representative Medications Used for the Management of Heart Failure With Reduced Ejection Fraction ${ }^{a}$

\begin{tabular}{|c|c|}
\hline Medication & Dose $^{t}$ \\
\hline \multicolumn{2}{|l|}{ Renin-Angiotensin Inhibitors } \\
\hline \multicolumn{2}{|l|}{ ARNI } \\
\hline Sacubitril/valsartan, mg twice per day & 200 \\
\hline \multicolumn{2}{|l|}{ ACEI } \\
\hline Enalapril, mg twice per day & $10-20$ \\
\hline Lisinopril & $20-40$ \\
\hline \multicolumn{2}{|l|}{ ARB } \\
\hline Losartan & 150 \\
\hline \multicolumn{2}{|l|}{ B Blocker } \\
\hline Metoprolol succinate & 200 \\
\hline \multicolumn{2}{|l|}{ MRA } \\
\hline Spironolactone & 50 \\
\hline
\end{tabular}

Abbreviations: $A C E I$, angiotensin-converting enzyme inhibitor; ARB, angiotensin receptor blocker; $\mathrm{ARNI}$, angiotensin receptor neprilysin inhibitor; MRA, mineralocorticoid receptor antagonist.

\section{Management of Comorbid Conditions and Lifestyle Modifications}

\section{Type 2 Diabetes}

One of the most common comorbidities associated with $\mathrm{HF}, \mathrm{T} 2 \mathrm{D}$ is found in $46 \%$ of patients hospitalized with acute decompensated HF. ${ }^{11}$ Although the initial target for T2D management was to prevent vascular events by lowering blood glucose levels, the concept of diabetes-related cardiomyopathy is now accepted. ${ }^{12,13}$ This concept includes the idea that most patients with T2D already have a component of cardiac involvement at the time T2D is identified, so primary prevention is not feasible. Therefore, HF preventive strategies need to be implemented earlier in individuals with T2D. ${ }^{13}$

Given this new paradigm of HF in the setting of T2D, a growing body of research now addresses the possibility that some agents used for the management of T2D may reduce cardiovascular mortality and hospitalization, whereas others may actually cause harm. Studies $^{14,15}$ of the sodium-glucose cotransporter 2 (SGLT-2) inhibitors have used large databases of national health care systems. The CVD-REAL 2 study (Comparative Effectiveness of Cardiovascular Outcomes in New Users of SGLT-2 Inhibitors) ${ }^{14}$ examined a broad range of cardiovascular outcomes

Table 2.

Description of Recommendations From the 2013 Guidelines for Management of Heart Failure With Reduced Ejection Fraction ${ }^{a}$

Size of Treatment Effect

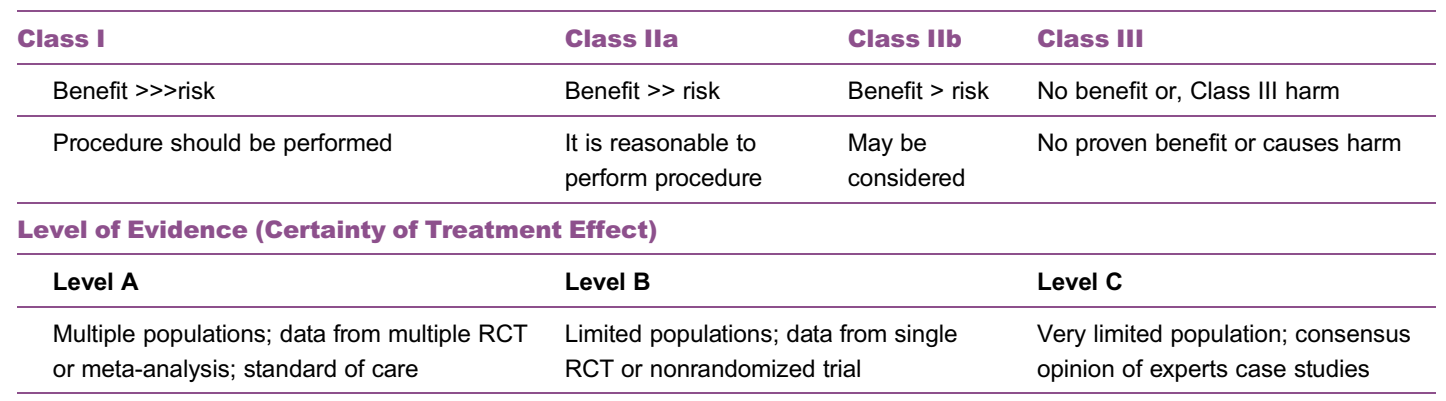

a Data are found in Yancy et al. ${ }^{1}$

Abbreviation: RCT, randomized control trial. 


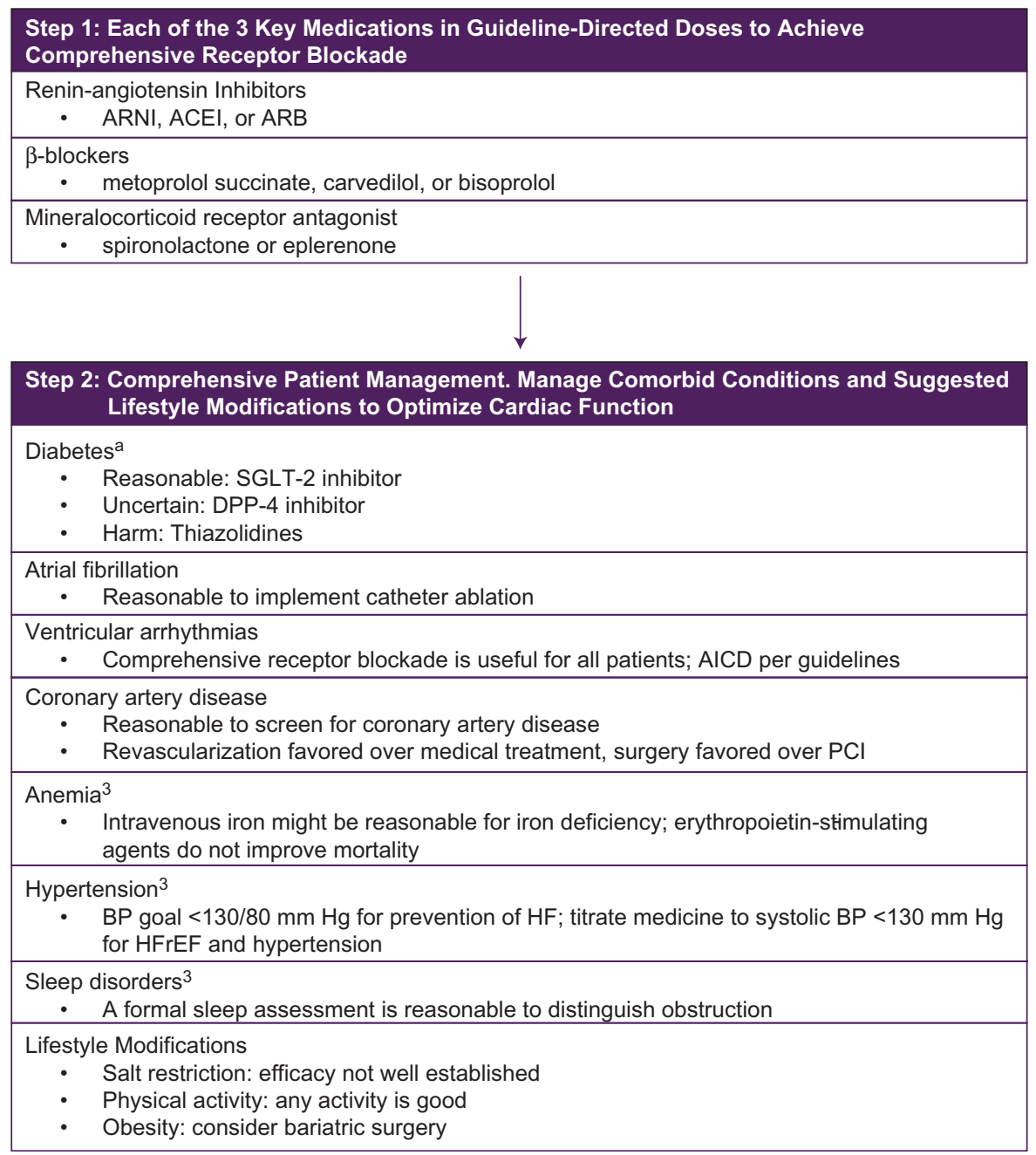

Figure 1.

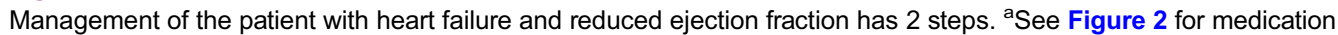
examples. Abbreviations: ACEI, angtiotensin-converting enzyme inhibitor; AICD, automatic implantable cardioverter-defibrillator; ARB, angiotensin receptor blocker; ARNI, angiotensin receptor-neprilysin inhibitor; BP, blood pressure; DPP-4, dipeptidyl peptidase-4; HF, heart failure; $\mathrm{HFrEF}$, heart failure with reduced ejection fraction; $\mathrm{PCl}$, percutaneous coronary intervention; SGLT-2, sodium-glucose co-transporter-2.

in a large international study of 6 countries totaling an initial 2.6 million patients receiving a new prescription for diabetic medications. The study group included 235,064 patients who were treated with an SGLT-2 inhibitor compared with an equal number of patients using other diabetic medications. A significant reduction was found in cardiovascular death and HF hospitalization in the group treated with SGLT-2 inhibitors.
In the Empagliflozin, Cardiovascular Outcomes, and Mortality in Type 2 Diabetes or the EMPA-REG OUTCOME trial, ${ }^{15}$ there was a $38 \%$ reduction in death and a $35 \%$ lower rate of hospitalization for HF.

In contrast, there is evidence of harm in terms of cardiovascular outcomes from several antihyperglycemic therapies. ${ }^{16}$ A randomized clinical trial ${ }^{16}$ suggested that metformin may reduce macrovascular 


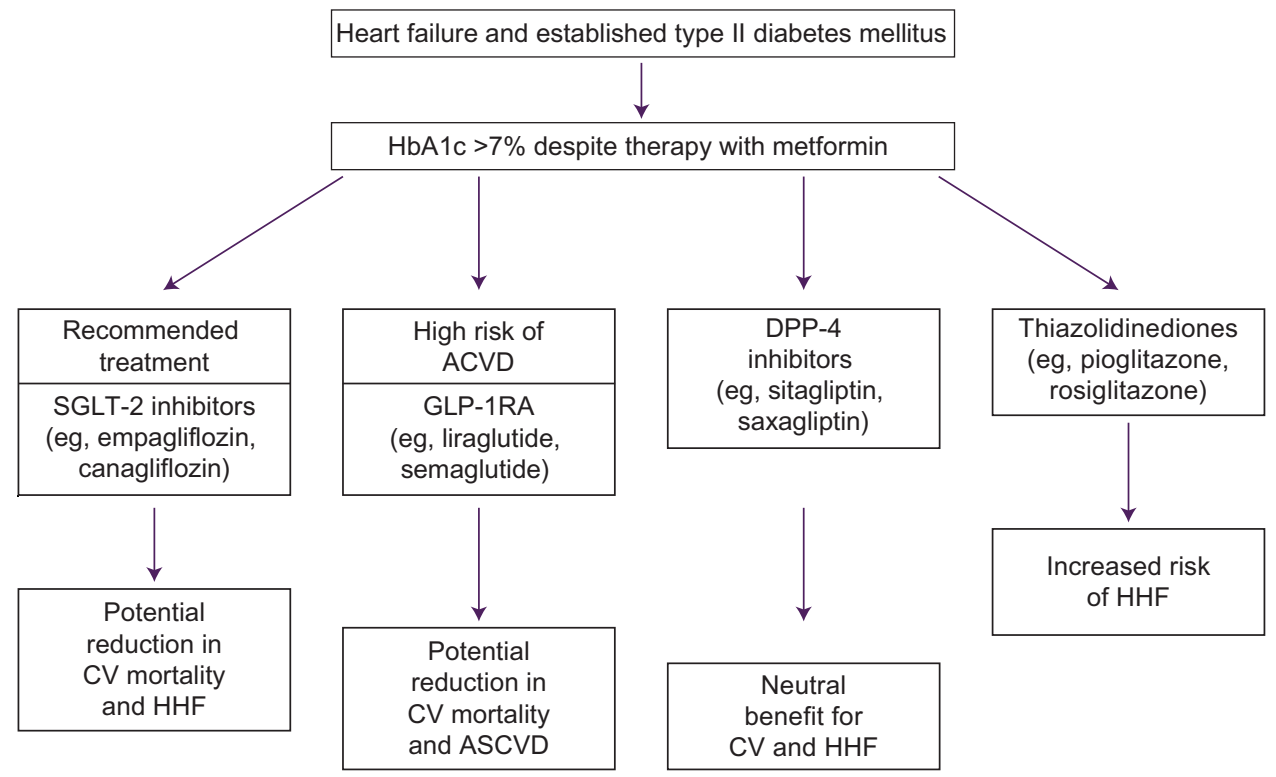

Figure 2.

Oral antihyperglycemic treatment strategy in patients with diabetes and heart failure with reduced ejection fraction. ${ }^{16}$ Abbreviations: ASCVD, atherosclerotic cardiovascular disease; CV, cardiovascular; DPP, dipeptidyl dipeptidase; GLP-1RA, glucagonlike peptide-1 receptor agonists; HbA1c, glycated hemoglobin; HHF, hospitalization for heart failure; SGLT-2, sodium glucose cotransporter.

events and is generally recommended as first-line treatment for patients with T2D. Several studies ${ }^{16-18}$ have suggested that thiazolinediones are associated with increased HF risk. Although some experts have warned against the danger of dipeptidyl-peptidase- 4 inhibitors, ${ }^{17}$ consensus statements suggest use with caution. Glucagonlike peptide-1 receptor agonists, such as liraglutide, have mixed reports on safety; a consensus statement would be that it has the potential for reduction in cardiovascular mortality, but it did not reduce risk for $\mathrm{HF}^{16,18}$

None of the studies of SGLT-2i agents categorize patients with HFrEF vs those with HF and preserved EF. Nonetheless, the preponderance of expert opinion recommends the use of SGLT-2i medications after lifestyle management and metformin to reduce major adverse cardiovascular events in patients with HFrEF. This opinion has been endorsed by the American Diabetes Association, and it is an approved indication by the Food and Drug Administration. ${ }^{19}$ We conclude that it is reasonable to initiate SGLT-2i in patients for whom lifestyle management and metformin have not decreased hemoglobin A1c effectively.

\section{Arrhythmias}

\section{Atrial Fibrillation}

The Framingham Heart Study ${ }^{20}$ described an increase in risk for HF in patients with atrial fibrillation, which has been confirmed in observations described in Schnabel et al. ${ }^{21}$ The proliferation of ambulatory monitoring devices now raises the hope that a threshold for atrial fibrillation duration might be defined and subsequently determine the risk for later HF. In the Asymptomatic Atrial Fibrillation and Stroke Evaluation in Pacemaker Patients and the Atrial Fibrillation Reduction Atrial Pacing Trial, or ASSERT, ${ }^{22}$ patients with hypertension aged 65 years or older with no history of atrial fibrillation were monitored with interrogation of an implantable pacemaker or defibrillator. Older age, greater body mass index, and longer subclinical atrial fibrillation duration within the first year were predictors of subclinical atrial fibrillation 
progression, which was independently associated with a 5-fold increase in the risk of hospitalization for HF. It is reasonable that patients with a pacemaker or defibrillator who demonstrate subclinical atrial fibrillation receive the best GDMT to prevent HF.

Because mortality and morbidity are higher among patients with $\mathrm{HF}$ and atrial fibrillation than with $\mathrm{HF}$ alone, the Catheter Ablation Versus Standard Conventional Therapy in Patients with Left Ventricular Dysfunction and Atrial Fibrillation or, CASTLE-AF, ${ }^{23}$ randomized 397 patients with paroxysmal or persistent atrial fibrillation to catheter-based pulmonary vein isolation or standard medical care for atrial fibrillation in addition to GDMT for HF. The study showed that pulmonary vein isolation in patients with HF was associated with a significantly lower rate of the composite end point of death from any cause or hospitalization for worsening HF than medical therapy. The 2019 ACC/ AHA focused update for the management of atrial fibrillation $^{24}$ concluded that it is reasonable to pursue catheter ablation for atrial fibrillation in patients with HFrEF.

\section{Ventricular Arrhythmias}

Patients with systolic HF progress to death for 1 of 3 reasons: (1) noncardiovascular comorbid conditions, (2) progressive pump dysfunction and worsening HF, or (3) sudden cardiac death. These factors may be linked. Severe left ventricular systolic dysfunction places patients with $\mathrm{HF}$ at high risk for sudden cardiac death. ${ }^{1,25}$ Although the initial impulse is usually to arrange consultation for an automatic implantable cardioverter/defibrillator (AICD) when a severe decrease in left ventricular ejection fraction (LVEF) is found, current recommendations emphasize GDMT to optimize receptor blockade as an essential step. ${ }^{26}$

The evidence for AICD in patients with HFrEF without evidence of ischemic heart disease is less strong than when CAD is present. In the Danish Study to Assess the Efficacy of ICDs in Patients with Nonischemic Systolic Heart Failure on Mortality, or the DANISH trial, ${ }^{27} 1116$ patients with systolic HF not caused by CAD were randomized to usual care or
AICD. At a follow-up of more than 5 years, death from any cause was $21.6 \%$ in the AICD group and $23.4 \%$ in the control group $(P=.28)$. Sudden cardiac death occurred in $4.3 \%$ in the AICD group and $8.2 \%$ in the usual care group.

In an editorial accompanying the report on the DANISH trial, McMurray ${ }^{28}$ pointed to the declining benefit of AICD over the past decade with the optimization of medical therapy for HFrEF, including the increased use of MRAs, the new agent sacubitril/valsartan, and coronary revascularization in patients with ischemic cardiomyopathy. A prospective crossover trial $^{29}$ of 120 patients with AICD and LVEF less than $40 \%$ compared appropriate use of defibrillation for ventricular tachycardia and nonsustained ventricular tachycardia over a period of 9 months when patients took ACEI or ARB compared with sacubitril/valsartan in the subsequent 9 months. The sacubitril/valsartan agent reduced sustained ventricular tachycardia and appropriate $\mathrm{AICD}$ shocks to $0.8 \%$ vs $6.7 \%$ in the group receiving ACEI or ARB.

The 2018 guidelines of the ACC/AHA and Heart Rhythm Society ${ }^{27}$ give a class IA recommendation to treat patients with HFrEF (LVEF <40\%) with a $\beta$-blocker, an MRA, and a renin-angiotonson inhibitor (either an ARNI, ACEI, or ARB) to reduce sudden cardiac death and all-cause mortality. The European and US guidelines recommend prophylactic ICD as a class I recommendation for patients with LVEF less than $35 \%{ }^{1,25}$

\section{Coronary Artery Disease}

In $49.5 \%$ of patients presenting to the hospital with acute decompensated HF, CAD is found as a comorbidity. ${ }^{11}$ In the general population, $50 \%$ to $65 \%$ of patients with HF have CAD. ${ }^{30}$ Patients with a first episode of $\mathrm{HF}$ should be screened for $\mathrm{CAD}^{1}$ and the utility of revascularization.

A retrospective cohort study ${ }^{30}$ reviewed records of more than 67,000 patients with new-onset HF during hospitalization. The principal finding was that $7.9 \%$ of patients received noninvasive testing for CAD while 
hospitalized, increasing to $14.6 \%$ by 90 days after admission.

The tests to screen for CAD are typically the same as in the general population. Traditionally, testing for myocardial viability was recommended before a decision for revascularization. However, the current evidence base for revascularization is based on a large-scale study ${ }^{31}$ in which patients did not undergo viability testing. If multivessel or left main CAD is found, the next step is to decide the best course of treatment. The Surgical Treatment for Ischemic Heart Failure, or STICH trial, ${ }^{31}$ demonstrated that coronary artery bypass graft (CABG) surgery provided a significant reduction in cardiovascular and overall mortality, as well as the need for rehospitalization compared with the patients randomized to medical therapy.

Do these conclusions hold true when percutaneous coronary intervention (PCI) is compared with medical therapy? A meta-analysis ${ }^{32}$ that included 2 studies involving 931 patients compared PCI with medical therapy. A statistically significant mortality reduction was observed with PCI. This meta-analysis then surveyed 16 studies that included 8782 patients with HFrEF and CAD, comparing CABG with PCI, and found a statistically significant reduction in mortality with CABG compared with PCI. ${ }^{32}$ Most of the patients did not undergo viability testing as part of their evaluation for $\mathrm{CAD} .^{32} \mathrm{We}$ conclude that it is reasonable to recommend $\mathrm{CABG}$ in patients with $\mathrm{HFrEF}$ and angina or decompensation of HF without viability testing.

\section{Lifestyle Modification}

The basic components of lifestyle modification to prevent and manage heart disease, including HF, and its comorbidities begin with 3 basic interventions: avoid tobacco in all forms, follow a nutritionally balanced diet, and stay physically active. ${ }^{33}$ For example, the Jackson Heart Study, ${ }^{34}$ a prospective observational study of 4129 black participants without a history of HF or CAD at enrollment showed that persons who smoked cigarettes had a significantly increased risk for left ventricular hypertrophy, systolic dysfunction, and incident HF hospitalization at a median follow-up of 8 years. Diets recommended for patients with heart disease have traditionally involved restriction of dietary sodium. However, data to support this approach for HFrEF are inconsistent. This controversy is manifested in the ACC/AHA guidelines. In 2013, the ACC/AHA guidelines downgraded the recommendation for sodium restriction to class IIa (reasonable) with $\mathrm{C}$ level of evidence. ${ }^{1}$ More specific information concerning sodium intake comes from the multihospital Heart Failure Adherence and Retention Trial, $^{35}$ which enrolled 902 patients with New York Heart Association class II and III HF and followed up with them for a median of 36 months. Sodium restriction was associated with a significantly higher risk of the composite end point of HF hospitalization or death. This increase was predominantly driven by hospitalization for HF; the increase in death was not statistically significant. ${ }^{35}$ A subgroup analysis suggested that patients were most likely to have increased risk if they were not receiving an ACEI or ARB. This hypothesisgenerating study ${ }^{35}$ represents a challenge to the conventional wisdom that dietary sodium restriction is a cornerstone in the management of HF.

Obesity has a moderately strong inverse relationship with HF outcomes. However, there is weak clinical trial evidence for modulating this comorbidity. ${ }^{4}$ This finding is complex because it has been reported that after PCI, patients with obesity had a better prognosis, with a lower incidence of HF. ${ }^{36}$ This obesity paradox has some nuance because body mass index does not discriminate between body fat and lean muscle. A study ${ }^{37}$ of 570 consecutive patients with HF demonstrated that arm circumference, a marker of muscle mass, predicted more accurately the favorable prognosis of patients with obesity and HF than abdominal girth, which is a marker of central obesity.

The Physician's Health Study ${ }^{38}$ demonstrated a beneficial effect of vigorous physical activity in all categories of baseline covariants, including weight, age, smoking, hypertension, and hyperlipidemia. Both skeletal muscle mass and muscle strength are protective 
against HF through a variety of physiologic mechanisms. ${ }^{37}$

The Physical Activity Guidelines for Americans ${ }^{39}$ issued by the US Department of Health and Human Services recommends that adults do at least 150 minutes of moderate-intensity exercise per week for substantial health benefits. The new guidelines recognize that adults with chronic conditions may not be able to meet this goal. In that case, they recommend that every adult "just move." 39

Obesity contributes to the development of HF through multiple mechanisms. However, the population of people with HF is no more successful at sustained weight loss through diet than the general population. Bariatric surgery provides long-term protection against the development of HF. ${ }^{40,41}$ A nonrandomized, prospective, controlled study ${ }^{42}$ of 2010 patients who were treated with bariatric surgery were compared with matched controls in Sweden. At a follow-up of 14.7 years, a significant reduction in cardiovascular deaths and cardiac events was found. A self-controlled study ${ }^{43}$ of 524 patients with HF had a reduced incidence of hospitalization or emergency department visits after bariatric surgery compared with obese patients who had other types of surgery. These preliminary data suggest that bariatric surgery can play a significant role in the management of obesity in patients with established HFrEF.

\section{Conclusion}

Currently, highly effective options exist for the management of HFrEF. However, a small fraction of patients with HFrEF are receiving the recommended medications at the recommended doses. Although computerassisted algorithms have improved outcomes in patients with acute coronary syndromes and peripheral artery disease, ${ }^{44}$ there is little progress in terms of improving the implementation of GDMT for HFrEF.

We propose a 2-step approach to HFrEF management. The first step is to ensure that the patient is taking 1 of each of the 3 key medication classes: (1) renin-angiotensin inhibitors (ARNI, ACEI, or ARB), (2) $\beta$-blockers, and (3) MRAs, in guideline-based doses to attain comprehensive receptor blockade. The second step is to address the comorbidities of HF, including T2D, atrial fibrillation, and CAD to fulfill comprehensive patient care. There is strong evidence for specific management of T2D, atrial fibrillation, and CAD that will reduce cardiac mortality and hospitalization. There is moderately strong evidence supporting lifestyle modifications, including weight loss through bariatric surgery for patients with obesity and physical activity for all patients. This report also described treatments that are of doubtful benefit (dietary sodium restriction) or may cause harm, such as certain drugs used to treat T2D.

\section{Acknowledgment}

We are grateful to Susan Householder, NP, for her thoughtful review and encouraging, helpful advice in manuscript preparation and to Colleen Streeter, MLIS, MPH, for her solid literature search support.

\section{References}

1. Yancy CW, Jessup M, Bozkurt B, et al. 2013 ACCF/AHA guideline for the management of heart failure: a report of the American College of Cardiology Foundation/American Heart Association Task Force on practice guidelines. Circulation. 2013;128:e240-327. doi:10.1161/ CIR.0b013e31829e8776

2. Yancy CW, Jessup M, Bozkurt B, et al. 2016 ACC/AHA/HFSA focused update on new pharmacological therapy for heart failure: an update of the 2013 ACCF/AHA Guideline for the management of heart failure. Circulation. 2016;134:e282-e293. doi:10.1161/ CIR.0000000000000435

3. Yancy CW, Jessup M, Bozkurt B, et al. 2017 ACC/AHA/HFSA focused update of the $2013 \mathrm{ACCF} / \mathrm{AHA}$ guideline for the management of heart failure: a report of the American College of Cardiology/American Heart Association Task Force on clinical practice guidelines and the Heart Failure Society of America. J Am Coll Cardiol. 2017;70:776-803. doi:10.1016/j.jacc.2017.04.025

4. Yancy CW, Januzzi JL Jr, Allen LA, et al. 2017 ACC expert consensus decision pathway for optimization of heart failure treatment: answers to 10 pivotal issues about heart failure with reduced ejection fraction: a report of the American College of Cardiology Task Force on expert consensus decision pathways [published correction appears in: J Am Coll Cardiol. 2018;72(20):2549. J Am Coll Cardiol. 2018;71(2):201-230. doi:10.1016/j.jacc.2017.11.025

5. Mozaffarian D, Benjamin EJ, Go AS, et al. Heart disease and stroke statistics-2016 update: a report from the American Heart Association. Circulation. 2016;133:e38-e360. doi:10.1161/CIR.0000000000000350 
6. Gawande A. The Checklist Manifesto: How to Get Things Right. New York, NY: Metropolitan Books; 2010.

7. Greene SJ, Butler J, Albert NM, et al. Medical therapy for heart failure with reduced ejection fraction: the CHAMP-HF Registry. J Am Coll Cardiol. 2018;72(4):351-366. doi:10.1016/j.jacc.2018.04.070

8. Butler J, Yang M, Manzi MA, et al. Clinical course of patients with worsening heart failure with reduced ejection fraction. J Am Coll Cardiol. 2019;73(8):935-944. doi:10.1016/j.jacc.2018.11.049

9. McMurray JJV, Packer M, Desai AS, et al. Angiotensin-neprilysin inhibition versus enalapril in heart failure. $N$ Engl $J$ Med. 2014;371:993-1004. doi:10.1056/NEJMoa1409077

10. Sangaralingham LR, Sangaralingham SJ, Shah ND, Yao X, Dunlay SM. Adoption of sacubitril/valsartan for the management of patients with heart failure. Circ Heart Fail. 2018;11(2):e004302. doi:10.1161/ CIRCHEARTFAILURE

11. Parikh KS, Sheng S, Hammill BG, et al. Characteristics of acute heart failure hospitalizations based on presenting severity. Circ Heart Fail. 2019;12(1):e005171. doi:10.1161/CIRCHEARTFAILURE.118.005171

12. Seferović PM, Paulus WJ. Clinical diabetic cardiomyopathy: a two-faced disease with restrictive and dilated phenotypes. Eur Heart J. 2015;36:1718-1727. doi:10.1093/eurhearti/ehv134

13. Farkouh ME, Verma S. Prevention of heart failure with SGLT-2 inhibition: insights from CVD-REAL. J Am Coll Cardiol. 2018;71 (22):2507-2510. doi:10.1016/j.jacc.2018.02.078

14. Kosiborod M, Lam CSP, Kohsaka S, et al. Cardiovascular event associated with SGLT-2 inhibitors versus other glucose-lowering drugs: the CVD REAL 2 study. J Am Coll Cardiol. 2018;71 (23):2628-2639. doi:10.1016/j.jacc.2018.03.009

15. Zinman B, Wanner C, Lachin JM, et al. Empagliflozin, cardiovascula outcomes, and mortality in type 2 diabetes. N Engl J Med. 2015;373 (22):2117-2128. doi:10.1056/NEJMoa1504720

16. Sharma A, Cooper LB, Fiuzat M, et al. Antihyperglycemic therapies to treat patients with heart failure and diabetes mellitus. JACC Heart Fail. 2018;6(10):813-822. doi:10.1016/j.jchf.2018.05.020

17. Packer M. Worsening heart failure during the use of DPP-4 inhibitors pathophysiological mechanisms, clinical risks, and potential influence of concomitant antidiabetic medications. JACC Heart Fail. 2018;6 (6):445-451. doi:10.1016/j.jchf.2017.12.016

18. Sattar N, Petrie MC, Zinman B, Januzzi JL Jr. Novel diabetes drugs and the cardiovascular specialist. J Am Coll Cardiol. 2017;69 (21):2646-2656. doi:10.1016/j.jacc.2017.04.014

19. Zelniker TA, Braunwald E. Cardiac and renal effects of sodium-glucose co-transporter 2 inhibitors in diabetes: JACC State-of-the-Art review. J Am Coll Cardiol. 2018;72(15):1845-1855. doi:10.1016/j. jacc.2018.06.040

20. Wang TJ, Larson MG, Levy D, et al. Temporal relations of atrial fibrillation and congestive heart failure and their joint influence on mortality: the Framingham Heart Study. Circulation. 2003;107(23): 2920-2925. doi:10.1161/01.CIR.0000072767.89944.6E

21. Schnabel RB, Rienstra M, Sullivan LM, et al. Risk assessment for incident heart failure in individuals with atrial fibrillation. Eur $\mathrm{J}$ Heart Fail. 2013;15(8):843-849. doi:10.1093/eurjhf/hft041

22. Wong JA, Conen D, Van Gelder IC, et al. Progression of device-related subclinical atrial fibrillation and the risk of heart failure. J Am Coll Cardiol. 2018;71(23):2603-2611. doi:10.1016/j. jacc.2018.03.519
23. Marrouche NF, Brachmann J, Andresen D, et al. Catheter ablation for atrial fibrillation with heart failure. N Engl J Med. 2018;378:417-427. doi:10.1056/NEJMoa1707855

24. January CT, Wann LS, Calkins $H$, et al. 2019 AHA/ACC/HRS focused update of the $2014 \mathrm{AHA} / \mathrm{ACC} / \mathrm{HRS}$ guideline for the management of patients with atrial fibrillation: a report of the American College of Cardiology/American Heart Association Task Force on Clinical Practice Guidelines and the Heart Rhythm Society in collaboration with the Society of Thoracic Surgeons. Circulation. 2019;139:e125-e151. doi:10.1161/CIR.0000000000000665

25. Ponikowski P, Voors AA, Anker SD, et al. 2016 ESC guidelines for the diagnosis and treatment of acute and chronic heart failure: the task force for the diagnosis and treatment of acute and chronic heart failure of the European Society of Cardiology developed with the special contribution of the Heart Failure Association. Eur Heart J 2016;37 (27):2129-2200. doi:10.1093/eurheartj/ehw128

26. Al-Khatib SM, Stevenson WG, Ackerman MJ, et al. 2017 AHA/ACC/ HRS guideline for management of patient with ventricular arrhythmias and the prevention of sudden cardiac death: executive summary: a report of the American College/American Heart Association Task Force on clinical practice guidelines and the Heart Rhythm Society. J Am Coll Cardiol. 2018;72:1677-1749. doi:10.1016/j. jacc.2017.10.053

27. Køber L, Thune JJ, Nielsen JC, et al. Defibrillator implantation in patients with nonischemic systolic heart failure. N Engl J Med. 2016;375(13):1221-1230. doi:10.1056/NEJMoa1608029

28. McMurray JJ. The ICD in heart failure-time for a rethink? N Eng J Med. 2016;375(13):1283-1284. doi:10.1056/NEJMe1609826

29. de Diego C, González-Torres L, Nuñez JM, et al. Effects of angiotensin-neprilysin inhibition compared to angiotensin inhibition on ventricular arrhythmias in reduced ejection fraction patients under continuous remote monitoring of implantable defibrillator devices. Heart Rhythm. 2018;15(3):395-402. doi:10.1016/j.hrthm. 2017.11.012

30. Doshi D, Ben-Yehuda O, Bonafede M, et al. Underutilization of coronary artery disease testing among patients hospitalized with new-onset heart failure. J Am Coll Cardiol. 2016;68(5):450-458. doi:10.1016/j.jacc.2016.05.060

31. Velazquez EJ, Lee KL, Deja MA, et al. Coronary artery bypass surgery in patients with left ventricular dysfunction. N Engl J Med. 2011;364 (17):1607-1616. doi:10.1056/NEJMoa1100356

32. Wolff G, Dimitroulis D, Andreotti F, et al. Survival benefits of invasive versus conservative strategies in heart failure in patients with reduced ejection fraction and coronary artery disease: a meta-analysis. Circ Heart Failure. 2017;10(1):e003255. doi:10.1161/ CIRCHEARTFAILURE.116.003255

33. Eyre H, Kahn R, Robertson RM, et al. Preventing cancer, cardiovascular disease, and diabetes: a common agenda for the American Cancer Society, the American Diabetes Association, and the American Heart Association. Circulation. 2004;109(25):3244-3255. doi:10.1161/01.CIR.0000133321.00456.00

34. Kamimura D, Cain LR, Mentz RJ, et al. Cigarette smoking and incident heart failure: insights from the Jackson Heart Study. Circulation. 2018;137(24):2572-2582. doi:10.1161/ CIRCULATIONAHA.117.031912

35. Doukky R, Avery E, Mangla A, et al. Impact of dietary sodium restriction on heart failure outcomes. JACC Heart Fail. 2016;4 (1):24-35. doi:10.1016/j.jchf.2015.08.007 
36. Gruberg, Weissman NJ, Waksman R, et al. The impact of obesity on the short-term and long-term outcomes after percutaneous coronary intervention: the obesity paradox? J Am Coll Cardiol. 2002;39 (4):578-584. doi:10.1016/s0735-1097(01)01802-2

37. Kamiya $\mathrm{K}$, Masuda $\mathrm{T}$, Matsue $\mathrm{Y}$, et al. Complementary role of arm circumference to body mass index in risk stratification in heart failure. JACC Heart Fail. 2016;4(4):265-273. doi:10.1016/j.jchf.2015.11.010

38. Kenchaiah S, Sesso HD, Gaziano JM. Body mass index and vigorous physical activity and the risk of heart failure among men. Circulation. 2009;119(1):44-52. doi:10.1161/CIRCULATIONAHA

39. 2018 Physical Activity Guidelines Advisory Committee. 2018 Physical Activity Guidelines Advisory Committee Scientific Report. Washington, DC: US Department of Health and Human Services; 2018. https:// www.health.gov/PAGuidelines. Accessed November 15, 2018.

40. Sundström J, Bruce G, Ottoson J, Marcus C, Näslund I, Neovius M. Weight loss and heart failure: a nationwide study of gastric bypass surgery versus intensive lifestyle management. Circulation. 2017;135 (17):1577-1585. doi:10.1161/CIRCULATIONAHA

41. Benotti PN, Wood GC, Carey, et al. Gastric bypass surgery produces a durable reduction in cardiovascular disease risk factors and reduces the long-term risks of congestive heart failure. J Am Heart Assoc. 2017;6(5):e005126. doi:10.1161/JAHA.116.005126

42. Sjöström L, Peltonen $M$, Jacobson $P$, et al. Bariatric surgery and long-term cardiovascular events. JAMA. 2012;307(1):56-65. doi:10.1001/jama.2011.1914

43. Shimada YJ, Tsugawa Y, Brown DFM. Bariatric surgery and emergency department visits and hospitalizations for heart failure exacerbation: population-based, self-controlled series. J Am Coll Cardiol. 2016;67:895-903. doi:10.1016/j.jacc.2015.12.016

44. White CJ. Cookbook medicine is the recipe for successfully managing patients with PAD. J Am Coll Cardiol. 2018;72(9): 1012-1014.

๑) 2020 American Osteopathic Association

\section{Earn CME Credits Online}

Many accredited online continuing medical education (CME) courses, including quizzes from The Journal of the American Osteopathic Association and its supplements, are available for physician-members of the American Osteopathic Association at https://minerva.osteopathic.org. 